\title{
Téoros
}

Revue de recherche en tourisme

\section{La restauration du futur}

\section{Joël Bourdeau}

Volume 3, numéro 3, novembre 1984

Cuisine québécoise, restauration et tourisme : vers une synergie féconde

URI : https://id.erudit.org/iderudit/1080781ar

DOI : https://doi.org/10.7202/1080781ar

Aller au sommaire du numéro

Éditeur(s)

Université du Québec à Montréal

ISSN

0712-8657 (imprimé)

1923-2705 (numérique)

Découvrir la revue

Citer cet article

Bourdeau, J. (1984). La restauration du futur. Téoros, 3(3), 30-32.

https://doi.org/10.7202/1080781ar

Ce document est protégé par la loi sur le droit d'auteur. L'utilisation des services d'Érudit (y compris la reproduction) est assujettie à sa politique d'utilisation que vous pouvez consulter en ligne.

https://apropos.erudit.org/fr/usagers/politique-dutilisation/
Cet article est diffusé et préservé par Érudit.

Érudit est un consortium interuniversitaire sans but lucratif composé de l’Université de Montréal, l'Université Laval et l'Université du Québec à Montréal. Il a pour mission la promotion et la valorisation de la recherche. https://www.erudit.org/fr/ 


\title{
La restauration du futur
}

\author{
par Joël Bourdeau *
}

II n'y a pas si longtemps, nous parlions de I'an 2000 comme d'une époque lointaine, et nous calculions l'âge que nous aurions au tournant du millénaire. Souvenez-vous aussi des recettes de science-fiction que nous lisions ici et lả: la pilule supervitaminée tiendrait lieu de repas, des chaines d'alimentation entièrement informatisées nous éviteraient les aléas d'un accueil ou d'un service souvent déficient. Encore 15 ans et nous y serons! Dans leur navette spatiale, les astronautes se mijotent des petits plats, et les expériences de cuisine sans intervention humaine ont échoué.

Pour avoir été souvent échaudés par le passé, de nombreux chefs d'entreprises placent la prospective au niveau de la boule de cristal ou du tarot. On lui reproche son verbiage, son manque de conclusions pratiques et ses errements. Notre monde est devenu très spécialisé et il s'avẻre difficile de regrouper tous les paramètres - démographiques, sociaux, économiques, etc. qui permettraient de prévoir le futur avec plus de précision. Aucun économiste sérieux n'ose s'aventurer au-delả de 5 ans, et mẻme pour cette échéance, les marges d'erreur s'avèrent parfois ènormes. Ainsi, tenter de prévoir la restauration de demain relève apparemment d'une gageure.

Dans I'industrie alimentaire, nous bénéficions cependant d'une certitude qui a fait ses preuves et qui devrait s'avérer juste pour quelques années encore: l'homme doit manger pour vivre. Fort de ce dogme, nos erreurs ne seront qu'accessoires et sans grand impact sur le fond. En effet, les modes de réalisation de ce besoin constituent des épiphénomènes liés a la société, à la culture et à l'économie dans lesquelles ils s'actualisent.

II est illusoire de vouloir examiner tous les aspects de la prospective alimentaire en si peu de pages. Aussi, nous attarderonsnous sur l'étude de quelques traits démographiques et socio-économiques ainsi que sur les impacts que ces tendances devraient créer au niveau de la restauration.

- Joel Bourdeau est technicien au Centre de recherches technologiques de IFI.T.H.Q.
Sans client, point de restauration; encore faut-ill savoir à qui s'adresse la cuisine que I'on concocte. Les besoins alimentaires d'un adolescent different de ceux d'une personne ågée. Les perspectives démographiques publiées par le Bureau de la statistique du Québec nous renseignent sur la composition de la sociétế de demain. Selon un scénario moyen, nous serons autour de 7 millions d'habitants en l'an 2006. La ventilation par groupe d'àge fait ressortir deux caractéristiques nouvelles dans la pyramide des ages. Les jeunes (de 0 à 14 ans) qui représentaient presque $30 \%$ de la population en 1971 ne compteront plus que pour $17 \%$. Par contre, le pourcentage des personnes âgées dans le même temps passera de $7 \%$ à $14 \%$ et ne cessera d'augmenter jusqu'en 2031.

Le vieillissement de la population résulte de deux facteurs: la baisse du taux de natalité, les démographes prévoyant un taux avoisinant $1 \% 111$, et l'amélioration de l'espérance de vie.

Signalons un autre phénomène récent: l'augmentation du nombre de personnes seules ou de ménages réduits. Les causes en sont diverses: divorces, jeunes qui quittent la maison pour vivre leur vie, mariages tardifs, décès du conjoint chez les personnes aggées.

Toutefois, l'extrapolation de cette tendance pour le futur doit se faire avec prudence. Certains indices encore bien fragmentaires semblent montrer que ce mouvement pourrait se ralentir, du moins en ce qui concerne les divorces, les départs des jeunes et les mariages tardifs.

Enfin, notons un autre facteur d'importance: la place de plus en plus importante des femmes dans la société. Une étude américaine prévoit qu'au toumant du siècle, $59 \%$ d'entre elles travailleront en dehors du foyer ${ }^{|2|}$. Nous crovons qu'au Québec, nous nous rapprocherons de ce chiffre.

Ces quelques prévisions qui paraissent, dans l'ensemble, assez vraisemblables pour un proche avenir, auront des incidences économiques importantes sur l'industrie de la restauration.

\section{Profil socio-économique}

De l'accroissement de la population et de son vieillissement découlent deux certitudes. II y aura 700000 bouches de plus à nourrir au Québec en l'an 2000. Par contre, pour un nombre égal, les personnes aggées mangent moins que les jeunes. Ceci compensant cela, la demande en nourriture ne s'accroitra pas beaucoup. La diminution du nombre de jeunes (et d'autres facteurs) amènera une perte de popularité de la restauration rapide, du moins telle qu'on la connaît aujourd'hui. En vieillissant, les consommateurs prendront conscience de la diététique et du caractère de fête du restaurant, deux aspects présentement absents de la restauration rapide. Leurs revenus augmentant avec l'äge, il sera possible a chacun de choisir un repas sans tenir compte des contraintes financières qui ne leur laissent souvent d'autre choix que le hamburger - frites. De plus, les adolescents prennent l'habitude de manger à l'extérieur du foyer et la garderont lorsqu'ils deviendront adultes. Ils constitueront bientôt le groupe $35-45$ ans, la clientèle idéale des restaurants.

Entre 1950 et 1980 , le pourcentage du budget alimentaire consacré au restaurant a connu une montée fulgurante. II est passé de 10 a $30 \%$. Depuis, it semble stagner autour de ce chiffre. La capacité de manger des gens n'est pas élastique. En fait, les dépenses per capita pour la nourriture ont légèrement baissé aux Ėtats-Unis entre 1970 et 1980 . Cette diminution serait due aux changements dans les habitudes alimentaires ${ }^{|3|}$. Conséquemment, l'augmentation des dépenses de restaurant ne se fait qu'aux dépens du budget alimentaire consacré au repas pris à la maison. La publicité devra donc convaincre le public qu'il est aussi avantageux de manger ả l'extérieur que chez-soi. Gráce aux données démographiques que nous évoquions précédemment, la démonstration devrait être aisée. En effet, la différence entre le coút d'un repas préparé à la maison et celui pris au restaurant s'amoindrit à mesure que le nombre de personnes diminue. 
Tableau 1

Pyramide des ages de la population du Québec au 1er juin 2006, scénario moven

Sexe masculin

Sexe féminin

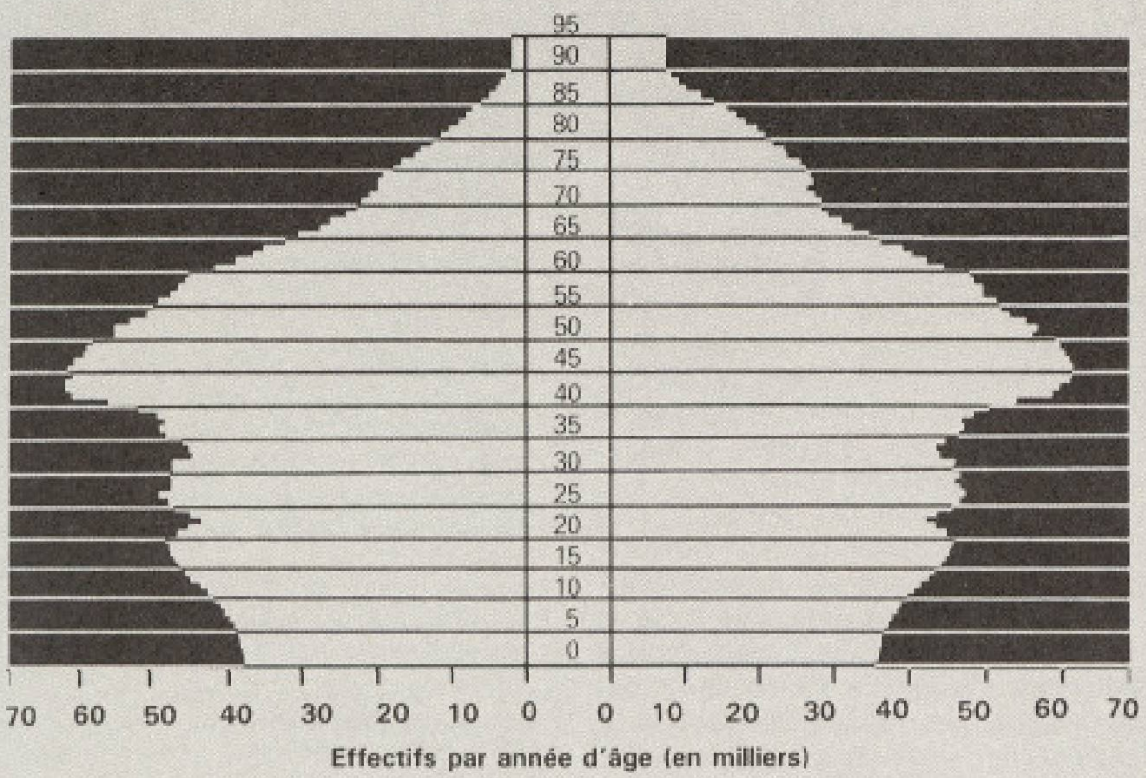

Source

Projections du Bureau de la statistique du Québec

Tableau 2

Structure de la population selon certains groupes d'äge. Québec, 1951-1981 ot 2006

0.14 15-39 $40.64 \quad 65+\quad$ Total Rapport de

dépendance

\begin{tabular}{|c|c|c|c|c|c|c|}
\hline 1951 & 1366108 & 1584089 & 873387 & 233107 & 4055691 & 0,650 \\
\hline$\%$ & 33,68 & 39,06 & 21,54 & 5,72 & 100,00 & \\
\hline 1961 & 1863395 & 1930525 & 1158990 & 306301 & 5259211 & 0,702 \\
\hline$\%$ & 35,43 & 36,71 & 22,04 & 5,82 & 100,00 & \\
\hline 1971 & 1785535 & 2389775 & 1439445 & 413005 & 6027760 & 0.574 \\
\hline$\%$ & 29,62 & 39,65 & 23,88 & 6,85 & 100,00 & \\
\hline 1981 & 1395735 & 2862715 & 1610585 & 569380 & 6438405 & 0,439 \\
\hline$\%$ & 21,68 & 44,46 & 25,02 & 8.84 & 100,00 & \\
\hline 2006 faible & 981221 & 2186915 & 2571470 & 968068 & 6707674 & \\
\hline$\%$ & 14,63 & 32,60 & 38,34 & 14,43 & 100,00 & 0,410 \\
\hline \multicolumn{7}{|l|}{2006} \\
\hline moyen & 1190971 & 2341070 & 2619650 & 975500 & $7127 \quad 191$ & \\
\hline$\%$ & 16.71 & 32,85 & 36,75 & 13,69 & 100,00 & 0,437 \\
\hline 2006 fort & 1362144 & 2431244 & 2656394 & 987013 & 7436795 & \\
\hline$\%$ & 18,32 & 32,69 & 35,72 & 13.27 & 100,00 & 0,462 \\
\hline \\
\hline \multicolumn{7}{|c|}{1951 à 1981: Recensements du Canada. } \\
\hline $2006:$ & Projections dt & Bureau de la & statistique du & Ouébec. & & \\
\hline
\end{tabular}

En d'autres termes, plus la famille est réduite, plus le restaurant s'avére avantageux.

Le travail des femmes a l'extérieur leur laisse peu de temps pour cuisiner à la maison, d'oú la tentation d'aller plus souvent manger au restaurant. Ce plaisir devient plus accessible grâce au revenu supplémentaire qu'elles apportent au ménage.

Le groupe de l'âge d'or, presque négligé jusqu'ici, devrait constituer une clientèle nouvelle pour l'hótelier et le restaurateur. Une enquête menée en 1981 par le Centre de recherches technologiques de l'Institut de tourisme et d'hötellerie du Québec auprès des personnes âgées, montrait que $25 \%$ de leur budget alimentaire passait au restaurant. Mème si on ne prévoit pas une augmentation significative de ce pourcentage, le marché demeure énorme grâce ả l'augmentation du nombre de personnes dans ce groupe d'áge in'oublions pas qu'elles seront 959000 en l'an 2001 .

\section{Les besoins}

Les besoins procèdent également des nouvelles données démogrpahiques. Les jeunes que nous formons aujourd'hui, et qui constitueront la plus grosse part du marché dans 15 ans, sont mieux éduqués, plus exigeants et plus conscients de la diététique. Ils demanderont moins la quantité que la qualité. La satisfaction plus que le prix importera, mais une satisfaction au moins égale au montant de l'addition. Afin de profiter au maximum de la vie, la santé deviendra une préoccupation plus importante qu'aujourd'hui,

Les personnes aggées dont personne ne s'occupe particulièrement aujourd'hui réclameront à leur tour quelques considérations. II faudra tenir compte de leurs besoins spécifiques, en alimentation bien sûr, mais également dans les aménagements - salle commune, jeux, éclairage, escalier, service-. Bien peu d'employés de restaurant comprennent la psychologie du troisième âge et son besoin d'attention et de gentillesse. L'amabilité du personnel de service est d'ailleurs le premier critère retenu par les personnes âgées pour retourner dans un restaurant

Le travail du couple modifiera la répartition des tăches domestiques: homme et femme se retrouveront à égalité devant la ucorvéew du souper. En effet, selon une étude réalisée aux Etats-Unis, $30 \%$ des hommes et $21 \%$ des femmes n'aiment pas cuisiner ${ }^{|4|}$. Sans pouvoir affirmer que ce pourcentage augmentera, il n'en demeure pas moins que ce chiffre représente un marché potentiel déjá énorme.

\section{Les tendances}

L'industrie alimentaire en général, et la restauration en particulier, devront donc répondre à ces besoins nouveaux. On peut 
prévoir que les grandes chaînes de restauration rapide qui suivent le marché de très près s'adapteront rapidement. Pendant que nous inventons la roborative poutine, les usines a hamburgers se recyclent dans la préparation du poulet et du poisson et installent des comptoirs à salades. II ne faudrait pas s'étonner de voir un jour McDonald se lancer dans la cuisine végétarienne ou macrobiotique.

Dans l'avenir, les restaurateurs devront proposer à leurs clients des menus diététiques et de régime, présenter pour chaque plat des informations sur le contenu en calorie, en sodium et en cholestérol, et offrir le choix de trois portions. Le temps des restaurants aattrape-tout $\omega_{\text {. }}$ qui proposaient un tour du monde culinaire tire à sa fin. L'ère de la spécialisation a ausși frappé la gastronomie. Cette industrie devra répondre aux besoins d'une société très fragmentée où les gens se regroupent plus par affinités électives que par classes sociales. Nous pourrons voir naitre ainsi des restaurants qui visent un créneau trẻs précis de la population, par exemple: les femmes d'affaires entre 35 et 45 ans, les kjoggersn, les écologistes, etc. Cette segmentation du marché aura pour conséquence de réduire la taille des restaurants. Les grandes salles, genre cafétéria de 300 places, disparaîtront, au moins pour le repas du soir. Cette tendance se fait déjà sentir dans la construction des hötels des grandes chailnes. Ils dépassent rarement 200 chambres.

Pour les personnes allergiques au fourneau qui ne veulent pas forcément prendre tous leurs repas au restaurant, nous devrions voir fleurir des établissement de amets à emporterw. Ceux-ci, judicieusement placés sur le chemin du retour à la maison, pourraient proposer une variété de plats, de l'entrée au dessert, faciles à réchauffer et à servir. Les restaurants qui jouissent d'une réputation gastronomique déjả établie proposeront leurs spécialités à emporter, fraiches ou congelées.

Et que mangerons-nous dans ces établissements à notre image? $\dot{A}$ ce propos, nous sommes et nous serons partagés entre deux aspirations contradictoires. D'une part, les moyens de communication ont fait du monde entier la banlieue de notre ville. aussi un méchoui, une paella ou un riz au cari ne nous dépaysent plus; par contre, lorsque nous voyageons, devant l'incohnu alimentaire, l'estomac s'angoisse et la vue d'une annonce de McDonald apparait comme une bouée de sauvetage là ce sujet, lire l'article intitulé Le voyage et /e repas). La palette alimentaire du futur oscillera donc entre ces dẹux extrèmes - le connu et l'inconnu - avec toutefois une propension à l'authenticité. Les plats régionaux très typiques feront ainsi contrepoids à l'uniformisation d'une restauration de masse.

\section{Et la technique?}

Fort de toute une tradition gastronomique, nous pouvons dire que les changements technologiques n'ont que peu affecté la façon dont les hommes satisfont leurs besoins alimentaires. Cette affirmation ne sous-entend pas que la technique n'a pas sa place dans la restauration, mais seulement qu'elle $n$ 'influence pas l'wexpérience» du repas en elle-même. toutes les tentatives pour remplacer les rapports interpersonnels ont échoué jusqu’á maintenant. $\mathrm{N}$ 'attendons done pas de grands changements dans ce sens, si ce n'est l'élimination de l'argent gräce à de nouvelles cartes de paiement. Les hôteliers s'adapteront pour offrir à leurs clients l'équivalent de ce qu'ils ont déjà chez eux. On peut donc s'attendre à une augmentation du nombre de chambres avec des terminaux d'ordinateurs, des machines de traitement de textes, des amodemsn. Les innovations les plus importantes demeureront cachées aux clients, et reléveront d'une saine administration: conservation de l'énergie par le recyclage des eaux usées et de la chaleur des cuisines, lavage de la vaisselle et douche par ultra-sons, nouvelle technologie du froid qui pourrait permettre le passage des aliments du congélateur à la table. Enfin. grâce à l'informatique, on peut prévoir que d'importantes firmes spécialisées prendront en charge la gestion des restaurants (comptabilité, achat, contrôle des stocks).

\section{Conclusion}

Notre prospective s'est volontairement limitée à imaginer l'impact démographique de trois catégories de personnes sur la restauration du futur: les jeunes, les femmes et les personnes ågées. Ses prémisses se fondent sur une extrapolation linéaire de ce que nous connaissons, c'est-â-dire une projection dans le temps des tendances actuelles. Bien que cette méthode soit courante, Alvin Toffler nous met en garde contre une telle démarche, car elle vest beaucoup plus fiable dans les périodes de stabilité que dans les périodes de bouleversements révolutionnaires comme celle que nous comaissons aujourd'huin (5).

Nous avons aussi délibérément omis de traiter des variables culturelles, psychologiques et politiques. Non qu'elles soient négligeables, au contraire, mais leurs prévisions font appel à tant de scénarios possibles qu'elles relévent somme toute de la divination.

Et puis! un futur sans mystere aurait-il encore de l'intérêt?

\section{Notes et références}

11) Ces prévisions pourraient s'avérer fausses. Il semble se dessiner chez les 20-35 ans en Californie un retour vers les vertus anciennes et les valeurs familiales.

121 Demographic Forecast: Labor force; American demographics, Fevrier 1981, page 47

13) LAWLESS, Mark et HART, Christopher; Forces that shape restawast demand. The Comell hotel and restaurant administration quarterly. Novembre 1980. page 14.

(4) Chain Marketing and Management Newsletter. 9 avril 1904, page 7 .

(5) TOFFLER, Alvin. Les cartes du futur. DeNoel 1983. page 225

\section{Bibliographie}

ANDERSON, Beverlee et LANGMEYLER, Lynn. The under and over 50 travelers dans Joumal of Travel Research. Printemps 1902.

BELAY, Jacques, Un manche loin d'atre satwe dans Né-restauration. Jamier 1984. Pages 20 a 25.

BRECH, Ronald, Projection to the year 2000 . That future for the hotel and catering industry? dans Adavances in catering technology. Applied Science. Publishers Ltd. Landon. 1980.

CANADA. Soixante cinq ans et plus. Rapport du conseil national du bien-être social sur les revenus des personnes ǵgées. Février 1904

Women more concemed than men about healthy eating. Chain marketing and management newslet. ter. Ayril 1904.

G.E, LIVINGSTON AND CHARLOTTE M. CHANG. Background and trends in carering to 1990 in the USA, dans Advances in catering technology. Applier Science. Publishers Lid. London. 1960. Pages, 16 a 39 .

PALMER, John. The meal of the futwe. Comell hotel and restaurant administration quarterly. Novembre 1984. Pages 7 \& 17

Taste of America. Restaurants and institutions. Decembre 19a3. Pages 105, 201

CANADIAN HOTEL and RESTAURANT Hospitality Market Report 1984-85. 